\title{
Hierarchical Sensor Networks: Capacity Bounds and Cooperative Strategies using the Multiple-Access Relay Channel Model
}

\author{
Lalitha Sankaranarayanan \\ WINLAB, Department of ECE \\ Rutgers University \\ Piscataway, NJ 08854 \\ Email: lalitha@winlab.rutgers.edu
}

\author{
Gerhard Kramer \\ Bell Laboratories \\ Lucent Technologies \\ Murray Hill, NJ 07974 \\ Email: gkr@bell-labs.com
}

\author{
Narayan B. Mandayam \\ WINLAB, Department of ECE \\ Rutgers University \\ Piscataway, NJ 08854 \\ Email: narayan@winlab.rutgers.edu
}

\begin{abstract}
A three-tier hierarchical wireless sensor network is considered that consists of a cluster of sensors, an intermediate relay with better computing and communication capabilities than the sensors, and a central server or access point. Such a network can be modeled as a multiple-access relay channel (MARC) with additive white Gaussian noise and fading. Capacity bounds for this network are presented with and without constraints on simultaneous reception and transmission by the relay. The results identify cooperative strategies between the relay and sensors for increasing network capacity. These strategies also preserve limited battery resources by eliminating the need for cooperation between sensors.
\end{abstract}

\section{INTRODUCTION}

Mechanical sensors of varying capabilities have been an integral part of automated systems since long before the birth of electronics. They tended, as did early electronic sensors, to be few in number and isolated, often application-specific, and carefully placed for maximal effectiveness. Now, a new generation of sensors is emerging with better sensing and communication characteristics and potentially important and varying uses. Advances in hardware have made it possible to design and manufacture compact multifunction sensors on silicon [1] with integrated radios and digital logic. While the size and affordable cost of these devices allow deployment in large numbers, their computing and communication capabilities can be exploited to form sensing networks whose integrated, analyzed information can be more valuable than that of an isolated sensor. The challenge lies in efficient use of these wireless sensors to form complex information gathering networks.

In their seminal work on the throughput of wireless ad hoc networks [2], Gupta and Kumar showed that for a network of $n$ homogeneous nodes that cooperate to forward data, the throughput per node falls asymptotically with increasing nodes as $\Theta(1 / \sqrt{n})$. The decrease in throughput at each node with increasing nodes is a direct result of interference limited communications and cooperation where each node gives up some of its available throughput to forwarding packets for neighboring nodes. This result prompts two important questions:

- Should every node in a sensor network participate in forwarding and relaying?

- Would the addition of a set of regularly distributed dedicated relay nodes with better processing and communication capabilities than the low-power sensor nodes change the scaling behavior of the per node throughput?

While it is hard to answer the first question in general, it is clear that in many sensing applications connectivity between all users is not required; the sensors merely observe and transmit data to those nodes with better routing and processing capabilities, and do not share data amongst themselves.

In [2] the authors show that addition of dedicated relays will not change the scaling properties if the relays use the same wireless channel. On the other hand, in [3] Liu et al consider a hybrid ad hoc network obtained by placing a sparse network of base stations (access points) connected by a highbandwidth wired network, within a random homogeneous ad hoc network of $n$ source nodes. Their results show that in such a network, where each source node transmits at $W$ bits/sec, a scaling in network throughput capacity as $\Theta(m W)$ can be achieved when the number of base stations, $m$, scales faster than $\sqrt{n}$ thus requiring a significant investment in the wired infrastructure.

Consider a three-tier hierarchical network [4] that results from the introduction of pure wireless relay nodes serving exclusively as forwarders in a hybrid wireless network of sources and base stations described above. Using the results of [2] and [3], it can then be expected that if the source nodes have a one-hop link to the nearest relay, and forwarding (cooperation) is limited to the relays, to first order, throughput scaling is achieved when the number of basestations exceeds $\sqrt{r}$, where $r<n$ is now the number of relays. Since now the relays forward data for all the sources, the requirement on the number of access points relative to the two-tier hybrid 


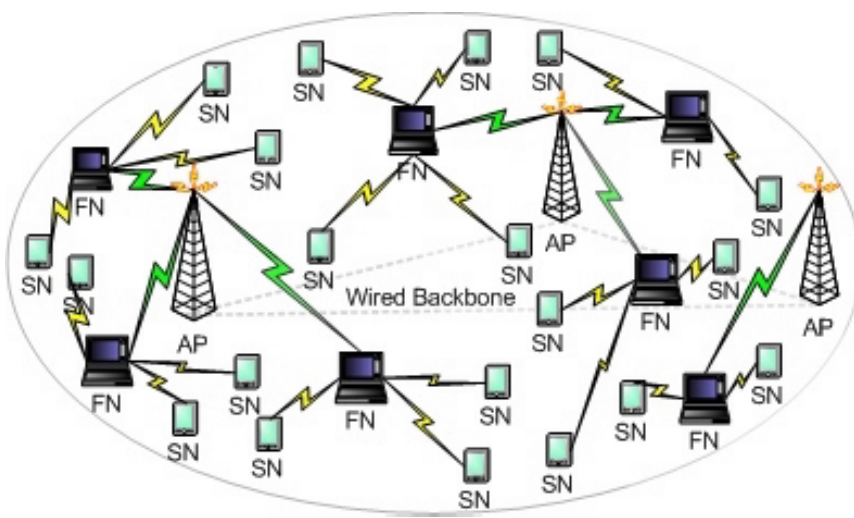

Fig. 1. A typical three-tier hierarchical network

network decreases by the factor equal to the square root of the ratio of the number of relays to the number of sources. Thus, while relays may not reverse the scaling behavior, they can help reduce the number of wired access points and also lower the power consumption of the source nodes, both valuable resources in a variety of sensor applications.

In addition to these general and theoretic networking issues, specifically for sensing applications, there are operational advantages to hierarchical heterogeneous layering that cannot be achieved with a "flat", homogeneous network of sensors, with its inherent limitations on power and processing capabilities. For instance, the relays help preserve limited battery resources of sensors by eliminating the need for sensors to monitor communications from their neighbors. In data gathering networks, the relay layer offers the advantage of caching and forwarding compressed data to the destination. Thus for a variety of applications, it appears that a relatively small number of higher-level network elements with access to more power and better computing and communication capabilities could greatly improve the performance of the overall system in terms of throughput, reliability, longevity, and flexibility. An example of such a three-tier hierarchical network is shown Fig. 1.

The results to date on the achievable throughput for sensor networks with and without hierarchy consider special cases of interference and communication strategies [5]. An analysis independent of such assumptions requires using the tools of information theory to determine capacity bounds for such a network. While network information theory is rich with open problems [6, chap. 14], for a simple hierarchical sensor network formed by a cluster of sensors communicating with a distant access-point (AP) via a forwarding node (FN) we can apply and extend several existing results to determine bounds on capacity, as well as construct capacity achieving strategies for certain geometries and channel models.

A three-tier hierarchical network resulting from a cluster of wireless sensors, a single forwarding node, and an access point can be modeled as a multiple-access relay channel (MARC) with Gaussian noise and fading. In section II we define the Gaussian MARC, review known capacity results for such a channel, consider two possible modes of relay operation that result from placing constraints on the simultaneous transmitreceive capabilities of the relay, and describe several cooperative communication strategies. In section III, we present capacity inner and outer bounds for both constrained and unconstrained MARCs. In section IV, we consider two example geometries for a two-sensor network and compare the performance of different communication strategies.

\section{Gaussian MARC: Model ANd StRategies}

\section{A. Model}

The multiple-access relay channel is a multi-source extension of the well-known single-user relay channel [7] and was introduced in [8] as a model for network topologies where multiple sources communicate with a single destination in the presence of a pure relay node. The paper [8] derived an upper bound on the capacity of a MARC by using a cut-set bound similar to [6, theorem 14.10.1]. This paper also presented an achievable rate region for the white Gaussian MARC.

A model for an $M$-source Gaussian MARC is shown in Fig. 2, and it consists of $M+1$ inputs signals $X_{k i}, k=$ $1,2, \ldots, M+1$ from the sources and the relay node, and two output signals $Y_{M+1, i}$ and $Y_{M+2, i}$ at the relay and destination, respectively, where $i$ is a time index. The channel is used $n$ times and the received signals at terminals $M+1$ and $M+2$ are

$$
\begin{aligned}
Y_{M+1, i} & =\left(\sum_{k=1}^{M} h_{M+1, k i} X_{k i}\right)+Z_{M+1, i} \\
Y_{M+2, i} & =\left(\sum_{k=1}^{M+1} h_{M+2, k i} X_{k i}\right)+Z_{M+2, i}
\end{aligned}
$$

where $Z_{j i}, j \in\{M+1, M+2\}$ is complex Gaussian noise with independent and indentically distributed (i.i.d) real and imaginary parts (circularly symmetric), that are zero-mean, and have variance $1 / 2$, that is, $E\left(\left|Z_{j i}\right|^{2}\right)=1$. The transmitted signals from the $k^{t h}$ source and the relay are constrained in power as

$$
\sum_{i=1}^{n} E\left(\left|X_{k i}\right|^{2}\right) / n \leq P_{k} \quad k=1,2, \ldots, M+1
$$

The parameter $h_{j k i}$ is the fading experienced by the signal from the $k^{t h}$ transmitter at the $j^{t h}$ receiver in the $i^{t h}$ symbol and is assumed known only at the $j^{\text {th }}$ receiver. In this analysis, analogous to the single-source case in [9], we consider two kinds of fading channels:

1) constant non-phase fading $h_{j k i}=1 / \sqrt{d_{j k}^{\gamma}}$ for all $i \in$ $[1, n]$ where $d_{j k}$ is the distance between the $j^{t h}$ receiver and the $k^{t h}$ source and $\gamma$ is the path-loss exponent. For this model, we define the source signal power as that 
received by the destination a unit distance away from the source.

2) ergodic phase-fading channel with parameter $h_{j k i}=$ $e^{\theta_{j k i}} / \sqrt{d_{j k}^{\gamma}}$ where $\theta_{j k i}$ is a uniformly distributed random variable between $[-\pi, \pi]$.

The analysis for these models generalizes to other types of fading such as Rayleigh fading [10].

The above model permits the relay to transmit and receive simultaneously [7]. For a wireless sensor network, this may be possible for certain geometries where the relay lies between the sensors and destination and uses directional antennas to transmit and receive thereby eliminating any interference between the two signals. In general, however, physical and practical constraints limit the relay to transmit or receive, thereby resulting in a constrained-MARC (C-MARC). Such relays have also been referred to as ‘cheap' relays [11].

We define a C-MARC to be a MARC where the relay receives for a fraction $\alpha$ of the total time and transmits for the remaining fraction $(1-\alpha)$. One can view this channel as having two states: the relay is in the "receive" state for $n_{1}$ symbols and in the "transmit" state for the remaining $n-n_{1}$ symbols, where $n_{1} / n=\alpha$. The output signals in the receive state for a Gaussian C-MARC are

$$
\begin{aligned}
Y_{M+1, i} & =\left(\sum_{k=1}^{M} h_{M+1, k i} X_{k i}\right)+Z_{M+1, i} \\
Y_{M+2, i} & =\left(\sum_{k=1}^{M} h_{M+2, k i} X_{k i}\right)+Z_{M+2, i}
\end{aligned}
$$

for $i \in\left[1, n_{1}\right]$ while those in the transmit state are

$$
\begin{aligned}
Y_{M+1, i} & =0 \\
Y_{M+2, i} & =\left(\sum_{k=1}^{M+1} h_{M+2, k i} X_{k i}\right)+Z_{M+2, i}
\end{aligned}
$$

for $i \in\left[n_{1}+1, n\right]$. The noise $Z_{j i}$ and the fading parameters $h_{j k i}$ are as defined for the MARC. In general, the two channel states of the Gaussian C-MARC need not occur in consecutive blocks, but for the following strategies there should be sufficiently many receive states before any transmit state.

We remark that an alternative definition for the C-MARC is to use the MARC of (1)-(3) and to simply add the constraints

$$
Y_{M+1}=0 \text { if } X_{M+1} \neq 0
$$

and that $X_{M+1}=0$ for a fraction $\alpha$ of the total time. The advantage of this approach is that one can apply the theory developed for the MARC directly to the C-MARC.

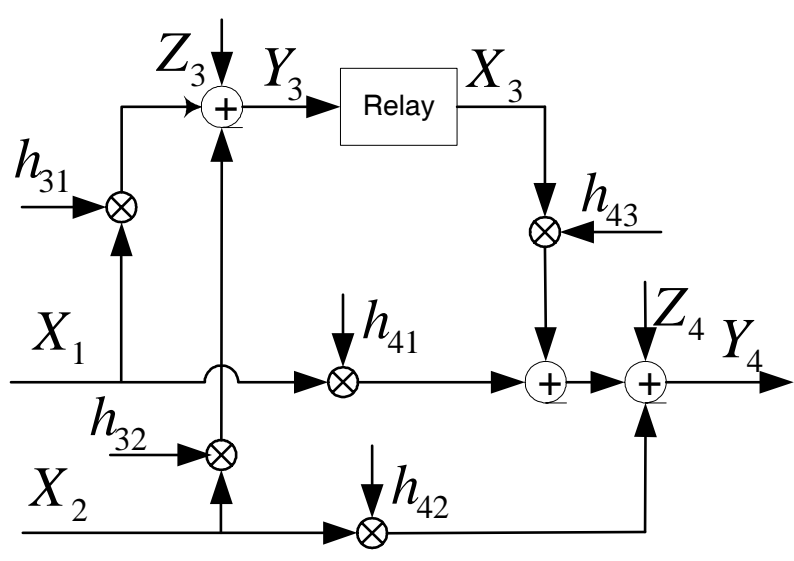

Fig. 2. A two-source Gaussian MARC

\section{B. Bounds and Cooperative Strategies}

A rate-tuple $\left(R_{1}, R_{2}, \ldots, R_{M}\right)$ is said to be achievable if there are encoders and decoders such that the probability that the destination node makes an error in decoding any of the $M$ messages is less than $\epsilon$ for all positive $\epsilon$. The capacity region is the closure of the set of achievable rate tuples. Outer bounds on the capacity region of the MARC and C-MARC can be obtained in a manner similar to the well-known cut-set bounds for networks. Inner bounds are obtained by constructing codes and computing their achievable rates.

Coding strategies for the classic single-source relay channel [7] can be extended to obtain various achievable rate strategies for the MARC. For example, one can generalize the cooperative strategy of [7, theorem 1] where the relay decodes the source messages before the destination and then cooperates with the source to aid the destination in decoding. This is known as the decode-and-forward strategy. This technique is also called information-theoretic multi-hopping [10] to highlight the fact that the source cooperates with the relay in addition to sending a new message and that the destination uses the signals received from both the source and relay to decode. The achievable rate region for a Gaussian MARC for the decode-and-forward strategy was presented in [8] and proved using regular block Markov encoding and backward decoding in [12]. In this paper, we extend the strategy of [7, theorem 6] where the relay facilitates reliable detection at the destination by forwarding a quantized version of its received signal to the destination. This results in a compressand-forward strategy for the MARC as the relay uses source coding techniques to quantize and forward its received signal from all $M$ sources to the destination. We also present an amplify-and-forward strategy for the Gaussian MARC as a multi-source extension of the strategy considered in [9] for the single-source Gaussian relay channel. Here the relay forwards an amplified version of its received signal from all sources to the destination subject to (3).

For the C-MARC, we present four achievable rate strategies. 
The cooperative decode-and-forward strategy is obtained when the relay decodes its received signal in the receive state and cooperates with the sources in the transmit state to aid the destination. If the sources, in addition to cooperating with the relay in the transmit state, transmit new messages directly to the destination, we obtain the cooperative partial decode-andforward strategy. For a single-source degraded and constrained relay channel, this strategy is included in [7, theorem 7] and achieves capacity [11]. Note, however, that our Gaussian channels are not degraded. We also present the compress-andforward strategy for the constrained MARC where the relay compresses its received signal from all sources in the transmit state and forwards it to the destination in the receive state. Finally, we consider an amplify-and-forward strategy for the Gaussian C-MARC for the special case of $\alpha=1 / 2$ as an extension of the amplify-and-forward strategy considered for the single-source constrained relay channel in [13]. However, unlike [13], we determine rate bounds by allowing the sources to transmit new messages in both fractions. The resulting rate regions for all strategies considered are presented in section III.

\section{SuMmARY OF RESUltS}

\section{A. MARC}

An outer bound for the MARC using cut-sets is obtained as follows. Let $G \subset S=\{1,2, \ldots, M\}$. Define $\mathbf{X}_{(G)}=\left\{X_{i}\right.$ : $i \in G\}, \mathbf{Y}=\left(Y_{M+1}, Y_{M+2}\right)$, and $G^{c}$ to be the complement of $G$ in $S$.

Theorem 1: The capacity region of an $M$-source MARC is a subset of the union $R_{M A R C}^{O B}$ of the sets of rate-tuples $\left(R_{1}, R_{2}, \ldots, R_{M}\right)$ satisfying

$$
\sum_{i \in G} R_{i} \leq \min \left\{\begin{array}{c}
I\left(\mathbf{X}_{(G)} ; \mathbf{Y} \mid \mathbf{X}_{\left(G^{c}\right)}, X_{M+1}, U\right), \\
I\left(\mathbf{X}_{(G)}, X_{M+1} ; Y_{M+2} \mid \mathbf{X}_{\left(G^{c}\right)}, U\right)
\end{array}\right\}
$$

for all $G \subset S$, where the union is over all input distributions $p\left(u, \mathbf{x}, x_{M+1}\right)=p(u) \cdot\left(\prod_{i=1}^{M} p\left(x_{i} \mid u\right)\right) \cdot p\left(x_{M+1} \mid u, \mathbf{x}\right), \mathbf{x} \triangleq$ $\left(x_{1}, x_{2}, \ldots, x_{M}\right)$ with $|\mathcal{U}| \leq 2^{M+1}-2$.

The cardinality bounds on the time-sharing random variable $U$ are obtained by applying the support lemma [14, pg. 310].

Consider the following achievable rate strategies.

1) Decode-and-Forward (DF): The rates achieved with this cooperative strategy were obtained in [10] as the set of rate tuples $\left(R_{1}, R_{2}, \ldots R_{M}\right)$ satisfying

$$
\sum_{i \in G} R_{i} \leq \min \left(\begin{array}{c}
I\left(\mathbf{X}_{(G)} ; Y_{M+1} \mid \overline{\mathbf{V}}, \mathbf{X}_{\left(G^{c}\right)}\right), \\
I\left(\mathbf{X}_{(G)}, X_{M+1} ; Y_{M+2} \mid \overline{\mathbf{X}}_{\left(G^{c}\right)}\right)
\end{array}\right)
$$

for all $G \subset S$ where $\overline{\mathbf{X}}_{\left(G^{c}\right)} \triangleq\left(\mathbf{V}_{\left(G^{c}\right)}, \mathbf{X}_{\left(G^{c}\right)}\right)$ and $\overline{\mathbf{V}} \triangleq$ $\left(\mathbf{V}_{(S)}, X_{M+1}\right)$. The auxiliary random variable $V_{i}$ enables cooperation between the $i^{\text {th }}$ source and the relay through the distribution $\left(\prod_{i=1}^{M} p\left(v_{i}\right) p\left(x_{i} \mid v_{i}\right)\right) \cdot p\left(x_{M+1} \mid \mathbf{v}\right)$ where $\mathbf{v}=$ $\left(v_{1}, v_{2}, \ldots, v_{M}\right)$. The first bound results from jointly decoding the source messages in each block at the relay while the second bound is obtained by decoding the cooperative information from the sources and relay at the destination. One might improve (8) by adding a time-sharing random variable as was done in theorem 1.

2) Compress-and-Forward (CF): Here the relay forwards a quantized version of its received signal from all sources to the destination. The destination first decodes the quantized signal $\hat{Y}_{M+1}$ from the relay and then uses $\hat{Y}_{M+1}$ and its received signal $Y_{M+2}$ to decode the source messages. The resulting region is the set of rate tuples $\left(R_{1}, R_{2}, \ldots R_{M}\right)$ such that

$$
\sum_{i \in G} R_{i} \leq I\left(X_{(G)} ; \hat{Y}_{M+1}, Y_{M+2} \mid X_{M+1}, \mathbf{X}_{\left(G^{c}\right)}\right)
$$

and

$$
I\left(X_{M+1} ; Y_{M+2}\right) \geq I\left(\hat{Y}_{M+1} ; Y_{M+1} \mid Y_{M+2}, X_{M+1}\right)
$$

where $X_{i}, i \in[1, M+1]$, are independent and $\hat{Y}_{M+1}$ is a probabilistic function of $Y_{M+1}$ and $X_{M+1}$. Observe that the source coding rate $I\left(\hat{Y}_{M+1} ; Y_{M+1} \mid Y_{M+2}, X_{M+1}\right)$, and hence the resulting distortion, is upper bounded by the rate $I\left(X_{M+1} ; Y_{M+2}\right)$ achievable between the relay and destination.

3) Amplify-and-Forward (AF): The amplify-and-forward strategy applies to Gaussian channels. The relay forwards an amplified version of its received signal as $X_{M+1, i}=$ $c Y_{M+1, i-1}$ where the constant $c$ is computed from (3). This strategy generates an inter-symbol interference (ISI) channel between the sources and destination and we obtain the rate region given by the multi-user water-filling algorithm of Cheng and Verdu [15]. We remark that, in addition to the assumption that the channel state information at each receiver is known only to that receiver, this strategy also requires knowledge at the destination of fading parameters at the relay.

\section{B. Constrained MARC}

Recall that the relay operates in a receive state and transmit state a fraction $\alpha$ and $1-\alpha$ of the time respectively. The channel state is modeled as known to all the $M+2$ nodes. An outer bound on the capacity can be derived by using cut-sets as follows. Let $\mathbf{X} \triangleq\left(X_{1}, X_{2} \ldots, X_{M}\right), \bar{\alpha} \triangleq 1-\alpha, \alpha \in[0,1]$, and $\overline{\mathbf{X}}_{(\cdot)} \triangleq\left(X_{M+1}, \mathbf{X}_{(\cdot)}\right)$.

Theorem 2: The capacity region of the $M$-source C-MARC is a subset of the union $R_{C-M A R C}^{O B}$ of the sets of rate tuples $\left(R_{1}, R_{2}, \ldots, R_{M}\right)$ satisfying

$$
\sum_{i \in G} R_{i} \leq \max _{\alpha} \min \left(\begin{array}{c}
\alpha I\left(\mathbf{X}_{(G)} ; \mathbf{Y} \mid \mathbf{X}_{\left(G^{c}\right)}, U\right) \\
+\bar{\alpha} I\left(\mathbf{X}_{(G)} ; Y_{M+2} \mid \mathbf{X}_{\left(G^{c}\right)}, U\right), \\
\alpha I\left(\mathbf{X}_{(G)} ; Y_{M+2} \mid \mathbf{X}_{\left(G^{c}\right)}, U\right)+ \\
\bar{\alpha} I\left(\overline{\mathbf{X}}_{(G)} ; Y_{M+2} \mid \mathbf{X}_{\left(G^{c}\right)}, U\right)
\end{array}\right)
$$

for all $G \subset S$, where the union is over all $p(u) \cdot \prod_{i=1}^{M} p\left(x_{i} \mid u\right)$ and $p(u) \cdot\left(\prod_{i=1}^{M} p\left(x_{i} \mid u\right)\right) \cdot p\left(x_{M+1} \mid u, x_{1}, x_{2}, \ldots, x_{M}\right)$ in the $\alpha$ and $(1-\alpha)$ fractions respectively with $|\mathcal{U}| \leq 2^{M+1}-2$.

As with theorem 1 , the cardinality bounds on the timesharing random variable $U$ are obtained by applying the support lemma [14, pg. 310]. 
Consider the following achievable strategies for the CMARC.

1) Decode-and-Forward (DF): This is similar to the DF strategy for the MARC except now the sources do not send a new message in the transmit state but simply cooperate with the relay to aid the destination in decoding the message sent in the receive state. The resulting bounds on the sum-rate for any set $G \subset S$ of the source indices are

$$
\sum_{i \in G} R_{i} \leq \max _{\alpha} \min \left(\begin{array}{c}
\alpha I\left(\mathbf{X}_{(G)} ; Y_{M+1} \mid \mathbf{X}_{\left(G^{c}\right)}\right), \\
\alpha I\left(\mathbf{X}_{(G)} ; Y_{M+2} \mid \mathbf{X}_{\left(G^{c}\right)}\right) \\
+\bar{\alpha} I\left(\overline{\mathbf{X}}_{(G)} ; Y_{M+2} \mid \mathbf{X}_{\left(G^{c}\right)}\right)
\end{array}\right)
$$

for independent $X_{i}, i \in[1, M]$, in both the transmit and receive states.

2) Partial Decode-and-Forward (P-DF): In addition to cooperating with the relay in the transmit state, the sources now directly transmit a new message to the destination. Then, the bounds on all combinations of the rate tuples for reliable detection at the relay and destination are

$$
\sum_{i \in G} R_{i} \leq \max _{\alpha} \min \left(\begin{array}{c}
\alpha I\left(\mathbf{X}_{(G)} ; Y_{M+1} \mid \mathbf{X}_{\left(G^{c}\right)}\right) \\
+\bar{\alpha} I\left(\mathbf{X}_{(G)} ; Y_{M+2} \mid \overline{\mathbf{X}}_{\left(G^{c}\right)}, \mathbf{V}\right), \\
\alpha I\left(\mathbf{X}_{(G)} ; Y_{M+2} \mid \mathbf{X}_{\left(G^{c}\right)}\right) \\
+\bar{\alpha} I\left(\overline{\mathbf{X}}_{(G)} ; Y_{M+2} \mid \hat{\mathbf{X}}_{\left(G^{c}\right)}\right)
\end{array}\right)
$$

where $\hat{\mathbf{X}}_{(\cdot)} \triangleq\left(\mathbf{V}_{(\cdot)}, \mathbf{X}_{(\cdot)}\right), \mathbf{V} \triangleq \mathbf{V}_{(S)}$, and $\left(V_{i}, X_{i}\right), i \in[1, M]$, are independent and $\mathbf{X} \rightarrow \mathbf{V} \rightarrow X_{M+1}$ forms a Markov chain.

3) Compress-and-Forward (CF): The relay quantizes the signal received in the receive state and forwards the appropriate quantized signal to the destination in the transmit state. Further in the transmit state the sources also send a new message directly to the destination resulting in a rate region

$$
\sum_{i \in G} R_{i} \leq \max _{\alpha}\left(\begin{array}{c}
\alpha I\left(\mathbf{X}_{(G)} ; \mathbf{Y}_{d} \mid \mathbf{X}_{\left(G^{c}\right)}\right) \\
+(1-\alpha) I\left(\mathbf{X}_{(G)} ; Y_{M+2} \mid \mathbf{X}_{\left(G^{c}\right)}\right)
\end{array}\right)
$$

for independent $X_{i}, i \in[1, M+1]$, subject to the constraint $\bar{\alpha} I\left(X_{M+1} ; Y_{M+2}\right) \geq \alpha I\left(\hat{Y}_{M+1} ; Y_{M+1} \mid Y_{M+2}\right)$ where $\hat{Y}_{M+1}$ is the auxiliary random variable used to represent the quantized signal at the relay and $\mathbf{Y}_{d} \triangleq\left(\hat{Y}_{M+1}, Y_{M+2}\right)$.

4) Amplify-and-Forward (AF): Similar to the unconstrained case, we set $X_{M+1, i}=c Y_{M+1, i-1}$ where in the $(i-1)^{t h}$ symbol the relay receives and in the $i^{t h}$ symbol it transmits. Clearly, this strategy only applies for the Gaussian C-MARC for the special case of $\alpha=1 / 2$. The resulting rate region is then a multiple-access extension of the single-user rate bounds obtained in [16] such that

$$
\sum_{i \in G} R_{i} \leq \frac{1}{2} I\left(\mathbf{X}_{(G)}^{1}, \mathbf{X}_{(G)}^{2} ; \mathbf{Y}_{M+2} \mid \mathbf{X}_{\left(G^{c}\right)}^{1}, \mathbf{X}_{\left(G^{c}\right)}^{2}\right)
$$

for some choice of $p\left(x_{(S)}^{1}, x_{(S)}^{2}\right)$ where $\mathbf{Y}_{M+2} \triangleq\left[Y_{M+2}^{1}\right.$ $\left.Y_{M+2}^{2}\right]^{T}$ is a vector of signals at the destination received in the first and second time-symbol while $\mathbf{X}_{\left(G^{c}\right)}^{j}=\left\{X_{i}^{j}: i \in G\right\}$ is the set of signals from the sources in $G$ transmitting in the $j^{\text {th }}$ time symbol, $j \in[1,2]$.

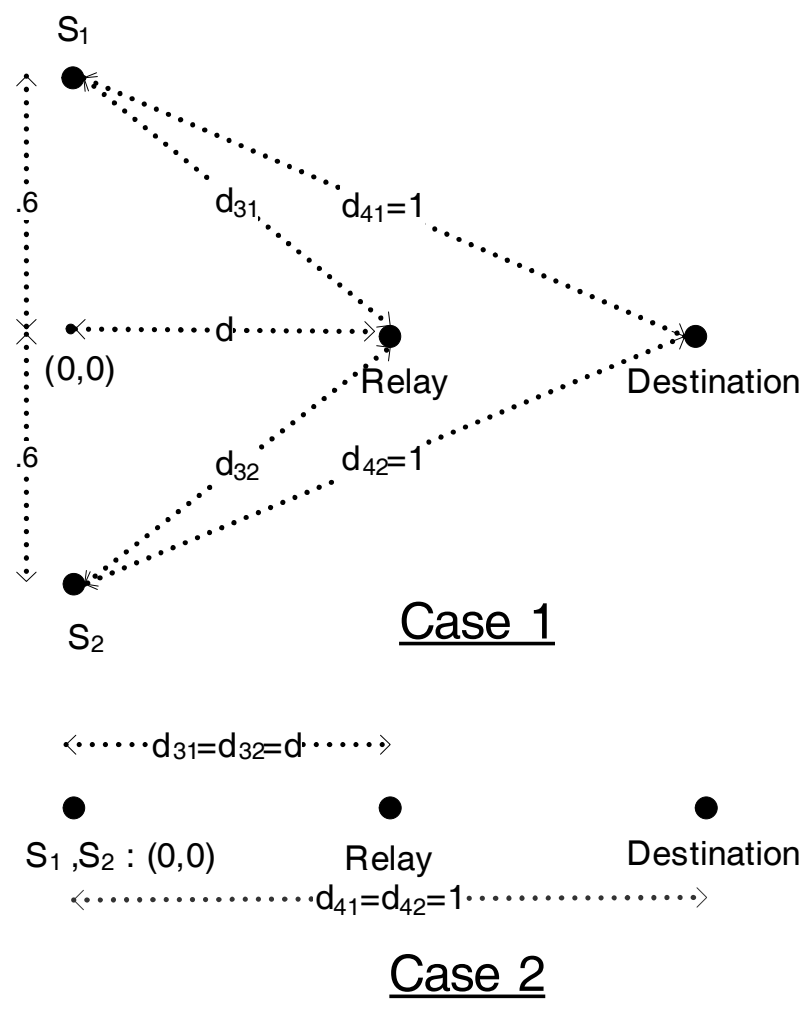

Fig. 3. Two geometries for a two-sensor MARC

\section{ILLUSTRATION OF RESUlts}

We consider the two example geometries shown in Fig. 3. While the two geometries chosen here clearly illustrate capacity achieving strategies, they are also reflective of the typical performance achieved by the various strategies considered here for an arbitrary placement of source and relay nodes. Case 1 is a geometry with a symmetric positioning of the sources with respect to the relay and destination while case 2 is a collinear geometry with both sources at the origin and the destination a unit distance away from the origin. In both cases, the relay moves along the line connecting the destination with the origin. We plot the two-sensor sum-rate in bits per channel use for each strategy as a function of the relay's distance from the origin. The transmitter signal-to-noise ratio (SNR) is chosen as $10 \mathrm{~dB}$ for both sources and the relay. We present and analyze the results separately for the two fading models we consider, namely the constant non-phase fading and the ergodic fast fading channel. For the following analysis we use the free-space path loss exponent $\gamma=2$ and evaluate all logarithms with respect to base 2 so that the resulting rates are in units of bits per channel use.

\section{A. Non-phase Fading}

1) MARC: The sum-rate of the three strategies, in addition to the outer bounds of theorem 1, are plotted in Figs. 4 and 5 for case 1 and case 2 respectively. The direct multiple-access 
sum-rate between the sources and destination is also plotted as a straight-line since it is independent of the relay's position.

For a $M$ source Gaussian MARC, the rate bounds for the DF strategy are maximized using Gaussian signaling [8] at the sources and relay such that

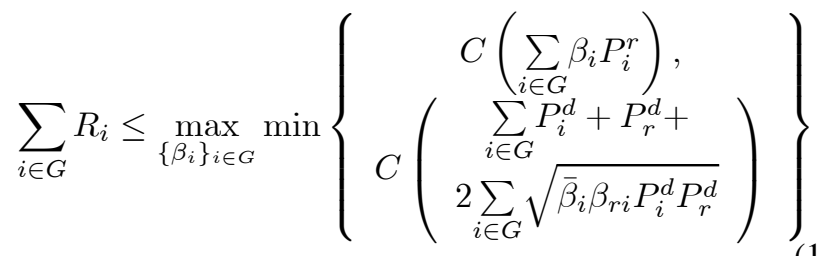

where $\beta_{r i}=\left(1-\beta_{i}\right) P_{i} / \sum_{i \in G}\left(1-\beta_{i}\right) P_{i}$ is the fraction of power allocated by the relay to cooperating with the $i^{t h}$ source, $\beta_{i}=\left(1-\bar{\beta}_{i}\right)$ is the fraction of power allocated by the $i^{t h}$ source to sending new messages, and $C(x) \triangleq \log (1+x)$. The corresponding source and relay signals are given as $X_{i}=\sqrt{\beta_{i} P_{i}} V_{i 0}+\sqrt{\bar{\beta}_{i} P_{i}} V_{i}$ for all $i \in[1, M]$ and $X_{M+1}=$ $\sum_{i \in G} \sqrt{\beta_{r i} P_{M+1}} V_{i}+\sqrt{\left(1-\sum_{i \in G} \beta_{r i}\right) P_{M+1}} V_{M+1}$ where $\left\{V_{i 0}\right\}_{i=1}^{M}$ and $\left\{V_{i}\right\}_{i=1}^{M+1}$ are i.i.d complex Gaussian circularly symmetric random variables with zero mean and unit variance. The quantities $P_{i}^{d}=P_{i} / d_{M+2, i}^{\gamma}$ and $P_{i}^{r}=P_{i} / d_{M+1, i}^{\gamma}$ are the power received from the $i^{\text {th }}$ source at the destination and relay respectively while $P_{r}^{d}=P_{M+1} / d_{M+2, M+1}^{\gamma}$ is the received power at the destination from the relay's signal. The plots also include the optimal fraction $\beta_{1}=\beta_{2}=\beta$, with the two fractions taking the same value $\beta$ for the symmetric geometry considered in case 1 and 2 at the maximum sum-rate point.

The two-source sum-rate bound for the CF strategy is obtained by constructing the quantized output as a noisy Gaussian signal subject to the constraint that the source coding rate that results from using side information at the decoder is bounded by the rate between the relay and destination. Similar to the DF strategy, the achievable bounds are obtained using Gaussian signaling in (14). Finally, the sum-rate bound for the AF strategy is obtained by determining the optimal multi-user water-filling power and rate allocation [15] for the case of symmetric sources (same received power and fading parameters at relay and destination for each source). We note that the notion of $\beta$ does not apply to the CF and AF strategies where the relay does not decode the source messages.

In Fig. 4, we observe that when the relay physically approaches the destination, the CF strategy approaches the upper bound achieving capacity when the relay and destination enjoy an error-free channel. This can be verified analytically [17] and results from the fact the distortion in the quantized signal decreases as the channel between the relay and destination becomes more reliable. Thus in the limit, the destination uses both channel outputs as if they were obtained from two receive antennas thus achieving the upper bound in (7). The AF strategy, similar to the single-user case [9], performs only as well as the DF strategy when the relay is very close to the

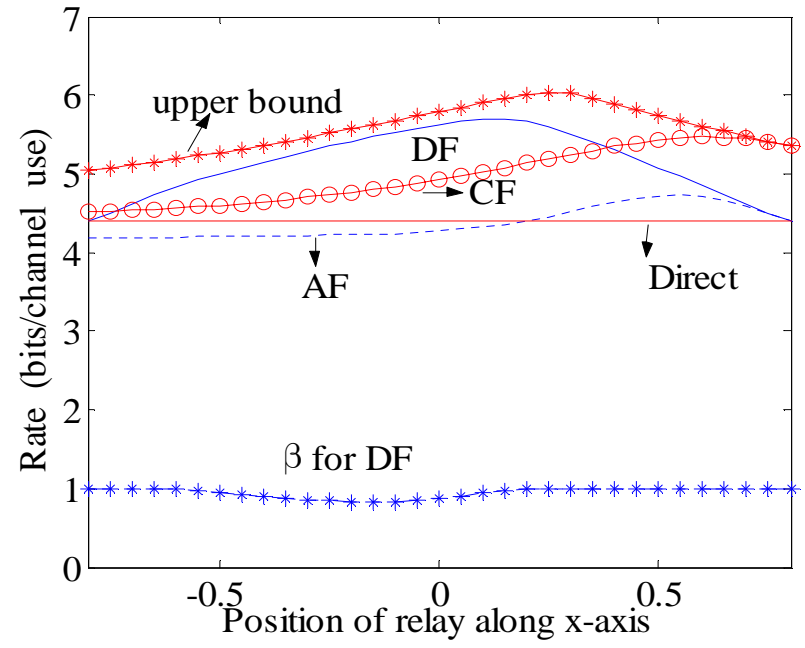

Fig. 4. Upper bound and achievable rates for the two-sensor MARC of Case 1 with $P_{1}=P_{2}=P_{r}=10 \mathrm{~dB}$

destination. The performance of the AF strategy suffers from the amplification of the received noise at the relay. Further as the relay moves away from the destination, the advantage of a high-rate channel between the relay and destination is also lost resulting in the AF strategy falling below the direct multipleaccess sum-rate that is achievable in the absence of the relay. The DF strategy on the other hand achieves its maximum when the relay is physically close to the two sources. The resulting high capacity channel between the relay and the sources forces maximum cooperation between the sources and the relay in aiding the destination decode. This is clear from the optimal $\beta$ curve in Figs. 4 and 5 for the DF strategy where $\beta=1$ (no cooperation) results only when the rate achieved at the destination exceeds the maximum rate possible between the sources and relay.

For the collinear geometry considered in case 2, we observe from Fig. 5 that the DF strategy approaches the upper bound when the relay is physically close to the two sources. It can be shown that the resulting channel when the relay and the sources are co-located is a physically degraded Gaussian MARC for which the DF strategy achieves capacity [17]. Finally, we note that the achievable rates for the DF and CF strategies are greater than the direct sum-rate when the relay is closer to the sources than the destination even if it does not physically lie between the sources and destination.

Thus, given a choice in the placement of the relay, a cooperative strategy can be chosen to achieve the best rate from among those strategies considered here.

2) Constrained MARC: The achievable sum-rate bounds for the two geometries of Fig. 3 are shown in Fig. 6 and 7. For the sake of simplicity and to make comparisons between the CF, DF, P-DF and AF strategies, we set $\alpha=1 / 2$. Analogous 


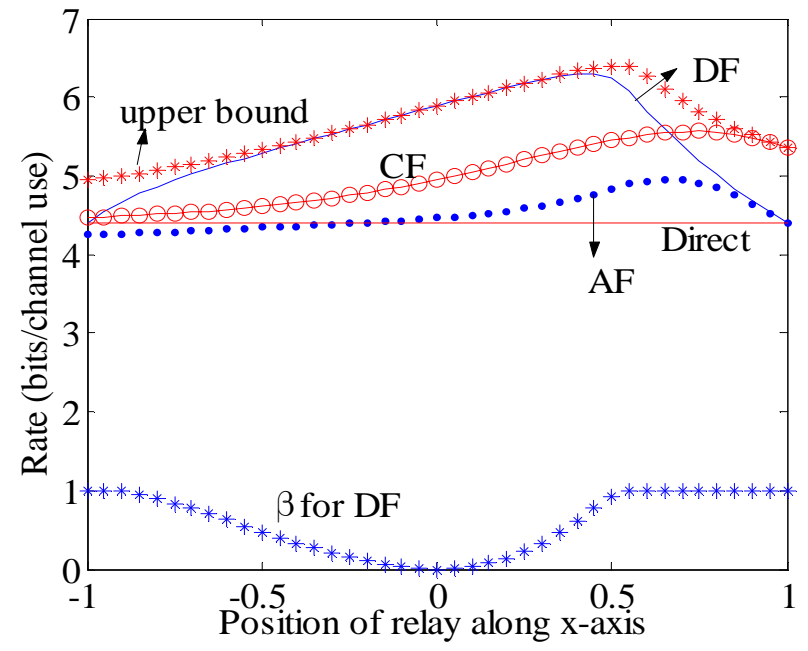

Fig. 5. Upper bound and achievable rates for the two-sensor MARC of Case 2 with $P_{1}=P_{2}=P_{r}=10 \mathrm{~dB}$

to the unconstrained case, for the Gaussian C-MARC, the achievable rate regions are maximized by appropriate choice of Gaussian signaling at the sources and relay for each strategy. Thus, for the DF and P-DF strategies, the transmitted signals in the fraction $\alpha$ are generated using an independent Gaussian random variable at each source. In the fraction $(1-\alpha)$, the cooperative DF strategy implies the relay cooperates with each source to transmit a sum of independent Gaussian signals while for the P-DF strategy, the signal design at the relay and sources is identical to that for the DF strategy for the MARC. Similarly, for the CF strategy, the quantized signal $\hat{Y}_{M+1}$ at the relay is constructed as a Gaussian distorted version of the received signal while the source signals are generated independently using Gaussian random variables.

As expected, for both cases the CF strategy approaches the upper bound as the relay approaches the destination. Here too, the CF strategy exploits the correlation between the received signals at the relay and destination (source coding with sideinformation at decoder) and for the case where the relay is at the destination, the destination has reliable access to both channel outputs thus achieving the capacity in (11). For case 2, it can be shown that a degraded Gaussian C-MARC results when the relay and sources are physically very close with both the P-DF and DF strategies achieving capacity [17] for this case. From Figs. 6 and 7, it is clear that except for the case where the sources and relay are very close to each other, the rate achievable by the DF strategy in (12) is smaller than that achievable by the P-DF strategy in (13) since the sensors in the DF strategy are strictly limited to cooperating with the relay in the $(1-\alpha)$ fraction. The optimal $\beta$ curve in Figs. 6 and 7 measures the fraction of power allocated to a new message at each source in the $(1-\alpha)$ fraction for the P-DF strategy. The sources do not cooperate with relay in the CF and AF strategies while for the DF strategy, $\beta_{1}=\beta_{2}=1$

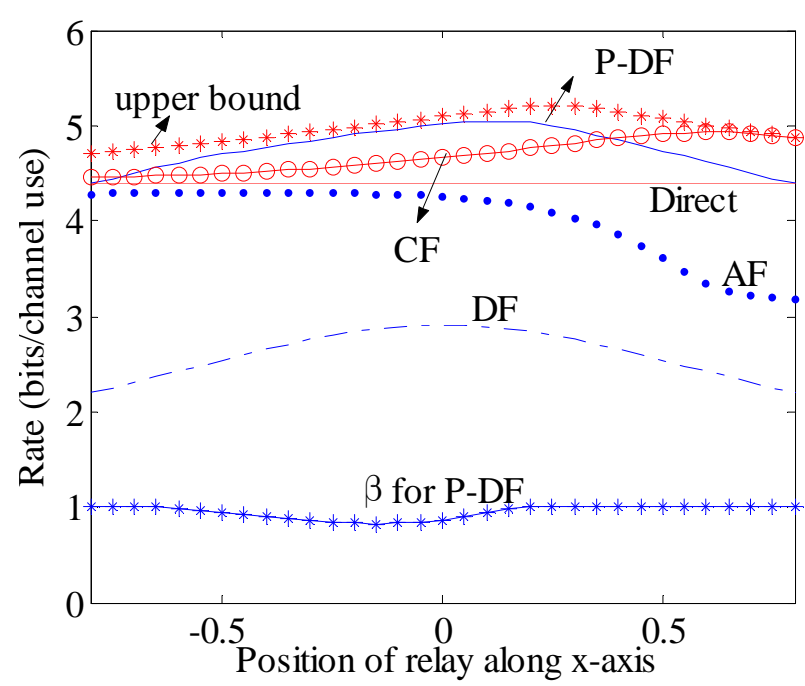

Fig. 6. Upper bound and achievable rates for the two-sensor C-MARC of Case 1 with $P_{1}=P_{2}=P_{r}=10 \mathrm{~dB}$

since the sources do not send a new message in the $(1-\alpha)$ fraction.

The AF strategy on the other hand has a performance worse than the direct sum-rate capacity. This is a direct result of the factor of $1 / 2$ that dominates the rate term in (15) in addition to the degradation in performance due to amplification of the received noise at the relay. So, for a more appropriate comparison, we compare the sum rates that result from limiting the source transmissions to every alternate time-symbol with and without the relay. With the relay, this results in the halfduplex amplify-and-forward strategy with the resulting rate region given by the set of rate tuples $\left(R_{1}, R_{2}, \ldots, R_{M}\right)$ such that

$$
\sum_{i \in G} R_{i} \leq \frac{1}{2} I\left(\mathbf{X}_{(G)}^{1} ; \mathbf{Y}_{M+2} \mid \mathbf{X}_{\left(G^{c}\right)}^{1}\right)
$$

for some choice of $p\left(x_{(G)}^{1}\right)$ where $\mathbf{Y}_{M+2}$ and $\mathbf{X}_{\left(G^{c}\right)}^{j}$ are as defined in (15). The advantage of using a relay when the sources are constrained to transmit only half the time is clearly demonstrated by comparing the rate achievable with and without the relay in Fig. 8 for both geometries. While the sum-rate achievable with the half-duplex AF strategy is smaller than that achievable with the AF strategy, this strategy may be advantageous when the sensors are limited in power and need to conserve their limited battery resources for extended use.

\section{B. Ergodic Phase Fading}

An ergodic phase fading model is useful to analyze channels that vary rapidly over all possible states within a message block. Such an analysis is appropriate for hierarchical sensor networks deployed over fast-changing terrain or in highmobility environments.

1) MARC: In [12], the authors show that for a MARC experiencing an ergodic fading channel as described in section 


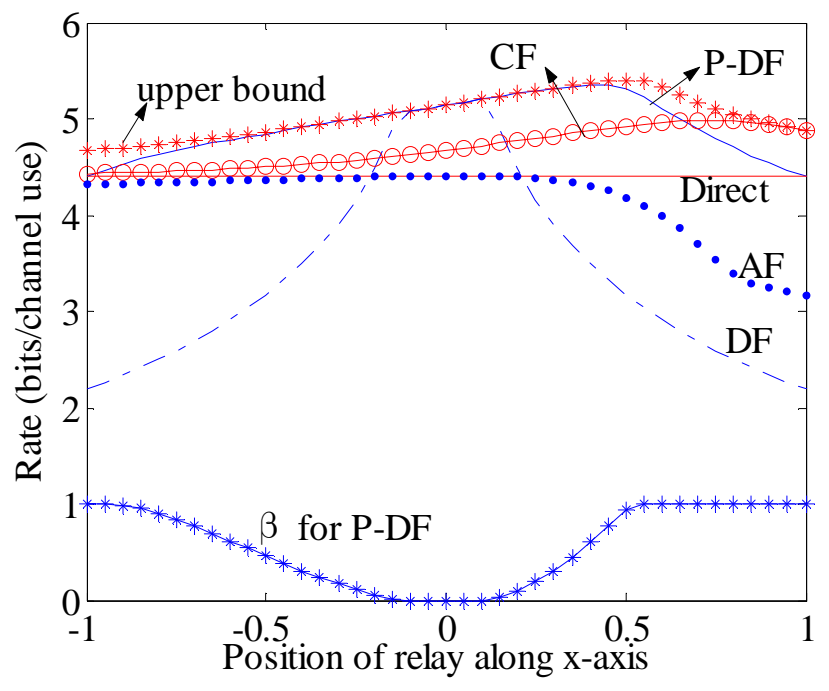

Fig. 7. Upper bounds and achievable rates for the two-sensor C-MARC of Case 2 with $P_{1}=P_{2}=P_{r}=10 \mathrm{~dB}$
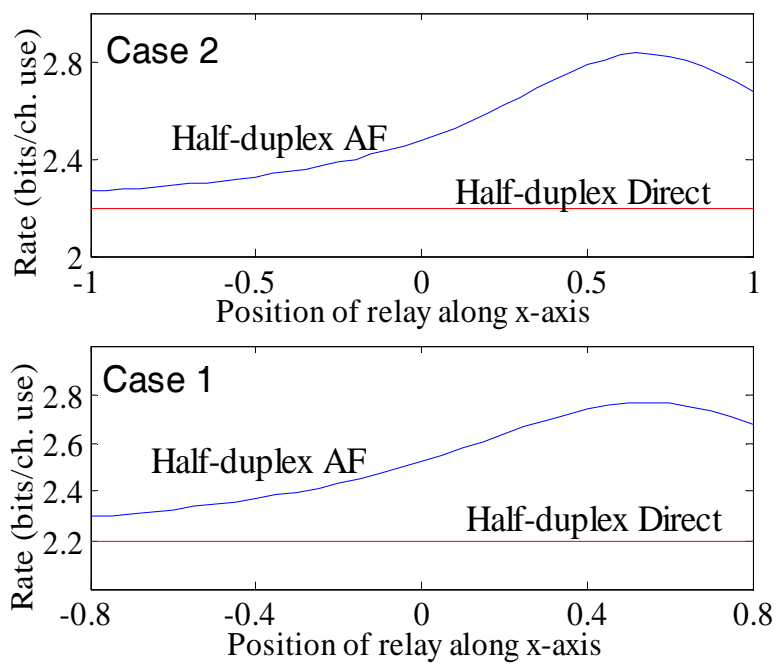

Fig. 8. Sum-rate achievable for the half-duplex AF strategy for Case 1 and 2 for $P_{1}=P_{2}=P_{r}=10 \mathrm{~dB}$

II, the decode-and-forward strategy achieves capacity when the relay lies in a region around the sources such that

$$
\left(\sum_{i \in G} \frac{P_{i}}{d_{M+2, i}}\right)+\frac{P_{M+1}}{d_{M+2, M+1}} \leq \sum_{i \in G} \frac{P_{i}}{d_{M+1, i}}
$$

is satisfied for all sets $G \subset\{1,2, \ldots M\}$.

The lack of channel state information at the transmitters coupled with a uniform phase-fading channel results in the ergodic capacity being maximized when the sources no longer cooperate with the relay in each block. The resulting rate region is then given by the set of $M$-tuples $\left(R_{1}, R_{2}, \ldots, R_{M}\right)$ that satisfy

$$
\sum_{i \in G} R_{i} \leq \min \left\{C\left(\sum_{i \in G} P_{i}^{r}\right), C\left(\sum_{i \in G} P_{i}^{d}+P_{r}^{d}\right)\right\}
$$
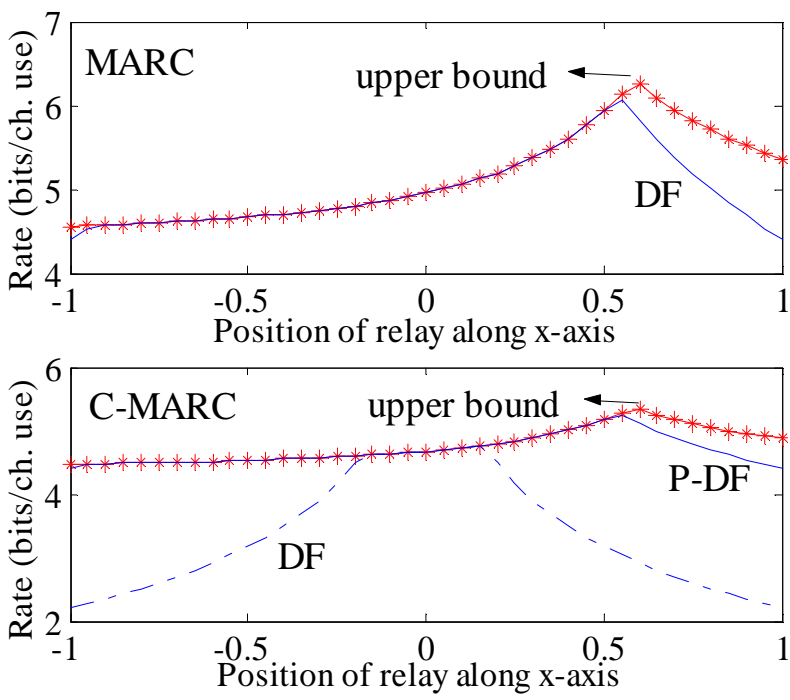

Fig. 9. Upper bound and Achievable Rates for the MARC and C-MARC of Case 2 under ergodic fading; $P_{1}=P_{2}=P_{r}=10 \mathrm{~dB}$

for all sets $G \subset\{1,2, \ldots M\}$ and $P_{i}^{r}, P_{i}^{d}$, and $P_{r}^{d}$ as defined earlier.

2) Constrained MARC: In an analogous manner, we can show that for the constrained MARC, the partial decode-andforward strategy achieves capacity when the relay lies in a region about the sources such that

$$
A^{(1-\alpha)}\left(\begin{array}{c}
\sum_{i \in G} \frac{P_{i}}{d_{M+2, i}} \\
+\frac{P_{M+1}}{d_{M+2, M+1}}
\end{array}\right) \leq\left(\begin{array}{c}
A^{\alpha}\left(\sum_{i \in G} \frac{P_{i}}{d_{M+1, i}}\right) \\
\cdot A^{(1-2 \alpha)}\left(\sum_{i \in G} \frac{P_{i}}{d_{M+2, i}}\right)
\end{array}\right)
$$

where $A^{\alpha}(x)=(1+x)^{\alpha}$. For the special case of $\alpha=1 / 2$, the above region simplifies to (18). The rate region for the CMARC under ergodic fading conditions is then given by the set of rate tuples $\left(R_{1}, R_{2}, \ldots R_{M}\right)$ such that

$$
\sum_{i \in G} R_{i} \leq \frac{1}{2} C\left(\sum_{i \in G} P_{i}^{d}\right)+\min \left\{\begin{array}{c}
\frac{1}{2} C\left(\sum_{i \in G} P_{i}^{r}\right), \\
\frac{1}{2} C\left(\sum_{i \in G} P_{i}^{d}+P_{r}^{d}\right)
\end{array}\right\}
$$

The capacity achieving behavior of the DF and P-DF strategy for the MARC and C-MARC respectively under ergodic fading conditions is clearly demonstrated in Fig. 9 for the collinear geometry of case 2 . The wide range of relay positions between $[-.9, .45]$ where the DF strategy achieves capacity for the MARC and between $[-.95, .45]$ for the C-MARC clearly illustrates how clustering the sources and relay helps achieve the ergodic capacity. Further for the C-MARC, the DF strategy also achieves capacity albeit over the smaller range $[-.15, .15]$ where the relay is very close to the sources. 


\section{CONCLusions}

We obtained capacity bounds for a three-tier hierarchical wireless sensor network using the Gaussian multiple-access relay channel model. An analysis for the constant non-phase and the ergodic phase-fading channel helped determine several achievable strategies for path-loss dominated and fast fading wireless channels. Notably for the two special geometries that result when either the sources and relay or the destination and relay form a very close cluster, we presented capacityachieving strategies for both the MARC and C-MARC. Further, for any general placement of relays and sources, a strategy that achieves the maximum rate can be chosen from among those presented here. The analysis can be extended to bound outage capacity, an appropriate performance metric for slow-fading channels typical of low-mobility and indoor environments. We can also extend the analysis to networks with multiple relays and access-points and use known results in information theory to better understand the differences between a flat network of cooperative nodes and a hierarchical ad hoc or sensor network using network capacity as the performance metric.

\section{REFERENCES}

[1] J. M. Kahn, R. H. Katz, and K. S. J. Pister, "Next century challenges: Mobile networking for "Smart Dust"," in ACM/IEEE Intl. Conf. on Mobile Computing and Networking (MobiCom 99), Seattle, WA, Aug. 1999.

[2] P. Gupta and P. R. Kumar, "The capacity of wireless networks," IEEE Trans. Inform. Theory, vol. 46, no. 2, pp. 388-404, Mar. 2000.

[3] B. Liu, Z. Liu, and D. Towsley, "On the capacity of hybrid wireless networks," in Proc. IEEE Infocom 2003, San Francisco, CA, Apr. 2003.

[4] S. Zhao, K. Tepe, I. Seskar, and D. Raychaudhuri, "Routing protocols for self organizing ad-hoc wireless networks," in IEEE Sarnoff Symposium 2003, Princeton, NJ, Apr. 2003.

[5] E. J. Duarte-Melo and M. Liu, "Data-gathering wireless sensor networks: organization and capacity," Computer Networks (COMNET): Special Issue on Wireless Sensor Network, vol. 43, no. 4, pp. 519-537, Nov. 2003.

[6] T. M. Cover and J. A. Thomas, Elements of Information Theory. New York: Wiley, 1991.

[7] T. Cover and A. E. Gamal, "Capacity theorems for the relay channel," IEEE Trans. Inform. Theory, vol. 25, no. 5, pp. 572-584, Sept. 1979.

[8] G. Kramer and A. J. van Wijngaarden, "On the white Gaussian multipleacess relay channel,” in Proc. IEEE Int. Symp. 2001, Sorrento, Italy, June 2000, p. 40.

[9] G. Kramer, P. Gupta, and M. Gastpar, "Capacity theorems for wireless relay channels," in Proc. 41st Annual Allerton Conf. on Commun., Control, and Computing, Allerton, IL, Oct. 2003.

[10] — - "Information-theoretic multi-hopping for relay networks," in International Zurich Seminar on Communications, ETH Zurich, Switzerland, Feb. 2004

[11] M. A. Khojastepour, A. Sabharwal, and B. Aazhang, "On the capacity of 'cheap' relay networks," in 37th Annual Conf. Information Sciences and Systems, Baltimore, MD, Mar. 2003.

[12] G. Kramer, M. Gastpar, and P. Gupta, "Cooperative strategies and capacity theorems for relay networks,” Feb. 2004, Preprint.

[13] J. N. Laneman, D. N. C. Tse, and G. Wornell, "Cooperative diversity in wireless networks: efficient protocols and outage behavior,” Apr. 2003, Preprint.

[14] L. Csisźar and J. Körner, Information Theory: Coding Theorems for Discrete Memoryless Systems. New York: Academic, 1981.

[15] R. S. Cheng and S. Verdu, "Gaussian multiple-access channels with intersymbol interference: Capacity region and multiuser water-filling," IEEE Trans. Inform. Theory, vol. 39, pp. 773-785, May 1993.
[16] R. U. Nabar, H. Bölcskei, and F. W. Kneubühler, "Fading relay channels: Performance limits and space-time signal design,” IEEE JSAC Special isue on Fundamental Performance Limits of Wireless Sensor Networks, 2003, accepted for publication.

[17] L. Sankaranarayanan, G. Kramer, and N. B. Mandayam, "Capacity theorems for the multiple-access relay channel," 2004, in preparation. 